\title{
A Complete Set of 22 Elementary Particles for an Expanded Standard Model
}

\author{
Daniel E. Friedmann \\ 2578 West 7 Avenue, Vancouver, Canada \\ Email:friedmann.daniel@alumni.ubc.ca
}

Received 29 April 2016; accepted 14 May 2016; published 17 May 2016

Copyright (C) 2016 by author and OALib.

This work is licensed under the Creative Commons Attribution International License (CC BY). http://creativecommons.org/licenses/by/4.0/

(c) (i) Open Access

\begin{abstract}
Our best understanding of how the known elementary particles and three of the forces (electromagnetic, weak and strong) are related to each other is encapsulated in the Standard Model of particle physics. However, the Standard Model is incomplete as it fails to explain a number of phenomena. The Standard Model relies on a set of elementary particles which are also known to be incomplete. This paper presents a complete set of elementary particles to serve as the basis for an expanded Standard Model. The set of elementary particles contains all known elementary particles and 6 extra elementary particles which relate only to mass and gravity. They include the graviton, a Higgs-like particle (but 6.13 times heavier than the Higgs), 2 dark matter particles and $\mathbf{2}$ dark energy particles for a total of 22 elementary particles (the $\mathrm{W}$ and $\mathrm{Z}$ bosons are seen as manifestations of a single particle).
\end{abstract}

\section{Keywords}

Elementary Particles, Standard Model, Beyond the Standard Model, Dark Matter Particles, Dark Energy Particles, Higgs

\section{Subject Areas: Particle Physics, Theoretical Physics}

\section{Introduction}

It is widely accepted by the scientific community that everything in the universe is made from several elementary particles and is governed by four fundamental forces. Our best understanding of how the known elementary particles and three of the fundamental forces (electromagnetic, weak and strong) are related to each other is encapsulated in the Standard Model of particle physics.

As successful as the Standard Model is, we know it's incomplete in several areas. For example: the Standard Model does not incorporate gravity; it does not deal with dark matter and energy particles; it does not explain why there are three levels of matter particles and it fails to provide a relationship between 19 parameters that 
must be derived experimentally, including the masses of most of the particles.

As the Large Hadron Collider (LHC) continues to produce more results we expect to see physics expand beyond the Standard Model. In fact, in late 2015 it's possible that a new particle in the $750 \mathrm{GeV}$ range was observed [1] [2]. Many proposals for models beyond the Standard Model, whether extensions (like supersymmetry) or new concepts (like string theory with its extra dimensions), have been proposed.

As the Standard Model sheds little light on the elementary particle masses, we have little guidance as to where to look for new particles.

This paper proposes a model for a complete set of elementary particles, including those not dealt with by the Standard Model. The Standard Model has 12 matter particles (that come in three generations of 6 quarks and 6 leptons), 4 force carrier particles ( 2 for the weak force) and the Higgs. The proposed set of elementary particles presented in this paper includes these particles, in addition to 6 other particles that relate only to mass and gravity. These 6 other particles include: the graviton (thought to be the force carrier for gravity), a Higgs-like particle but 6.13 times heavier than the Higgs, 2 dark matter particles and 2 dark energy particles, for a total of 22 particles (as will be shown in this proposed model the $\mathrm{W}$ and $\mathrm{Z}$ bosons governing the weak interaction are seen as manifestations of a single entity).

The complete set of 22 elementary particles is derived from a manuscript [3] dating back to at least the first century, which categorizes all building blocks of the universe in detail. This manuscript is purely a theoretical construct, as opposed to the Standard Model which has been built by advancing theories and confirming them experimentally.

The author understands that it is unacceptable to use these kinds of manuscripts in order to motivate new theories. Nonetheless, the manuscript does shed much light on what is already known and does predict additional particles not found in the Standard Model. In addition, most of the extensions of the Standard Model are motivated by a belief in unification and simplicity, also without any scientific source, but in fact, in agreement with the source text of the manuscript relied upon herein.

\section{The Complete Set of 22 Elementary Particles}

The complete set of 22 elementary particles in the proposed model is shown in Figure 1. In the manuscript [4] the model is derived by interconnecting 10 channels, which are arranged in three columns, in all possible ways. These connections naturally lead to 3 horizontal connections, 12 diagonal connections and 7 vertical connections. In total, the connections represent the 22 building blocks of nature (which correspond to the 22 Hebrew letters).

Figure 1 consists of 10 circles connected by 22 lines. Each of the 22 lines individually represent an elementary particle - denoted by a Hebrew letter. Each Hebrew letter has a name, symbol, value and a simple value as indicated in Table 1. The value denotes the number that each Hebrew letter represents, and the simple value denotes the sequential number of the letter in the Hebrew alphabet.

We note the following characteristics of the 22 particle proposed model depicted in Figure 1:

1) There are 3 letters along horizontal lines (horizontal particles), there are 12 letters along diagonal lines (diagonal particles), and there are 7 letters along vertical lines (vertical particles).

2) Horizontal particles. The 3 horizontal particles [5] carry the 3 non-gravitational forces: a) the Shin $\boldsymbol{w}$, the electromagnetic; b) the Aleph $\aleph$, the weak; and c) the Mem $n$, the strong force. These non-gravitational forces do not interact with the vertical particles, only with the diagonal particles. Note that the Aleph $\mathrm{x}$ is a composite of two letters (corresponding to the $\mathrm{W}$ and $\mathrm{Z}$ bosons) as will be explained in Section 3, entitled Letter Shapes and Particles.

3) Diagonal particles. There are 12 diagonal particles [6] in 3 levels of 4 particles each, indicated within each of the dark boxes in Figure 1. At each level there are 2 particles on the left-side-associated with constraint and bound, and 2 on the right-side-associated with unconstraint. The particles on the left-side are the quarks at each of the 3 energy levels, and the particles on the right-side are the leptons at each energy level.

4) Vertical particles. There are 7 vertical particles [7] that emanate in 3 pillars. The middle pillar contains the mass and gravity related particles. At the bottom, the Tav $ת$ is the carrier of the gravitational force [8] or graviton. The next particle upward, the Resh $\urcorner$, is the Higgs, and the top particle, the Dalet 7 , is a Higgs-like particle but heavier. In the left-side pillar are the 2 dark matter particles. As is the case for the left-side diagonal particles, these particles are heavy and constrained and are thus what scientists have been calling dark matter. In the right-side pillar are the lighter unconstrained particles and are thus what scientists have been calling dark energy 


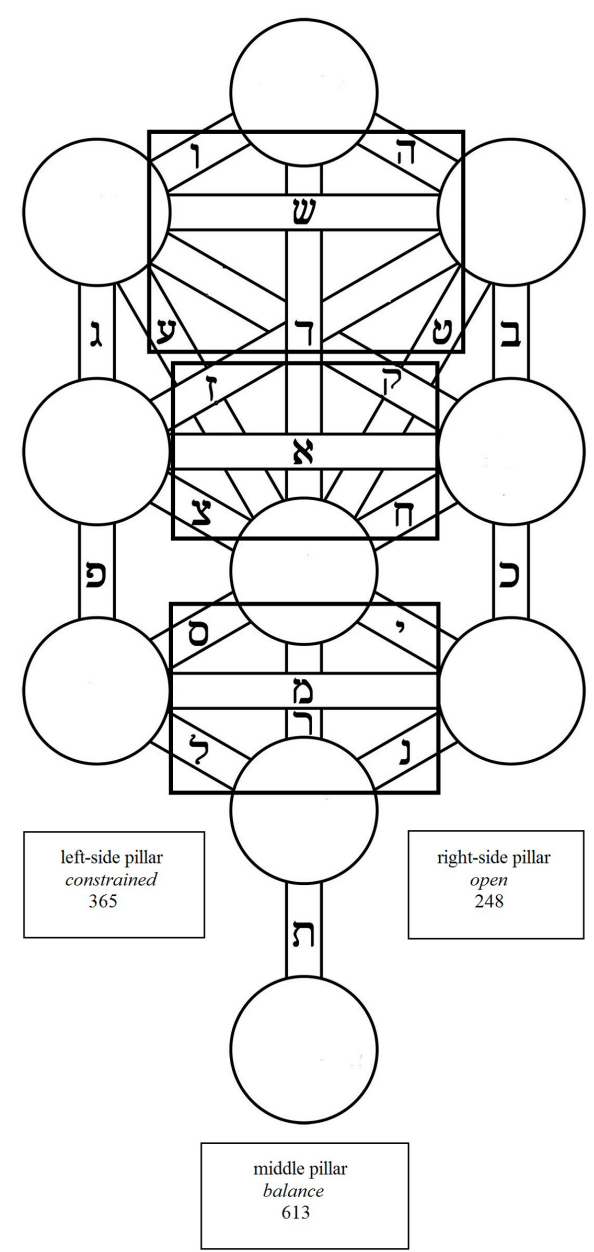

Figure 1. The complete set of 22 elementary particles.

Table 1. Hebrew letter names and values.

\begin{tabular}{|c|c|c|c|c|c|c|c|c|}
\hline Name & Alef & Bet & Gimel & Dalet & Hey & Vav & Zayin & Het \\
\hline Symbol & $\kappa$ & $ב$ & $\lambda$ & $T$ & ה & I & $i$ & $\pi$ \\
\hline Value & 1 & 2 & 3 & 4 & 5 & 6 & 7 & 8 \\
\hline Simple value & 1 & 2 & 3 & 4 & 5 & 6 & 7 & 8 \\
\hline Name & Tet & Yud & Kaf & Lamed & Mem & Nun & Samesh & Ayin \\
\hline Symbol & 0 & , & כ & $ל$ & D & J & 0 & $y$ \\
\hline Value & 9 & 10 & 20 & 30 & 40 & 50 & 60 & 70 \\
\hline Simple value & 9 & 10 & 11 & 12 & 13 & 14 & 15 & 16 \\
\hline Name & $\mathrm{Pe}$ & Tsadi & Kuf & Resh & Shin & Tav & & \\
\hline Symbol & פ & צ & P & ר & ש & ת & & \\
\hline Value & 80 & 90 & 100 & 200 & 300 & 400 & & \\
\hline Simple value & 17 & 18 & 19 & 20 & 21 & 22 & & \\
\hline
\end{tabular}

particles. These vertical particles occur only at the second and third energy levels (note that the dark boxes identify energy levels for diagonal particles only) with the exception of the graviton at the first energy level (represented by the Tav $ת$ ). 
5) Horizontal and vertical axis are independent. Thus, vertical particles do not interact via horizontal particles, i.e., via the 3 non-gravitational forces, they only interact with the force of gravity. Diagonal particles interact with horizontal and vertical forces, i.e., all forces and also vertical mass particles in the middle pillar (Higgs and Higgs-like).

6) The right-side, left-side and middle pillars are associated with the pillar's numbers of 248, 365 and 613, respectively ${ }^{1}$.

7) Each energy level has a particular scale factor which, when multiplied by the Hebrew letter value, determines the particle's mass, as will be shown in Section 4, entitled Particle Masses-some predictions. a) To determine the scale factor of a higher energy level one multiplies the scale factor of the lower energy level by the associated pillar number then divides by 2, e.g., by 365/2 for the left-side pillar. b) To correlate scale factors at the lowest level on the right-side with the left-side pillars one simply multiplies the scale factor by the ratio of the pillar numbers; thus to go from scale factor on the right-side pillar to the scale factor on the left-side pillar one multiplies by $365 / 248$. c) To correlate the scale factors at the lowest level on the middle pillar with the right-side pillar one multiplies the right-side pillar lowest level scale factor by 40 ; the value of the letter Mem $\Delta$ connecting the lowest levels.

8) Each Hebrew letter shape reveals an underlying reality and will be illustrated in Section 3, entitled Letter Shapes and Particles.

\section{Letter Shapes and Particles}

The Hebrew alphabet consists of 22 letters. Each Hebrew letter has 3 modalities; a pictogram, a letter and a number. Each of these modalities provides underlying information about it as a building block of nature. We examine the three horizontal force particles and their associated letter shapes, for illustration purposes.

1) Electromagnetic force: The Shin $w$ has 3 heads and is actually pronounced differently when a dot is placed over the rightmost or leftmost head. These two ways to pronounce the letter Shin $w$ correlate to polarizations of the photon.

2) The Strong force: the Mem $\Delta$ actually comes in 2 shapes-the $1^{\text {st }}$ shape (shown in Table 1 ) is used when the letter Mem appears anywhere but at the end of the word. The $2^{\text {nd }}$ Mem shape $\square$, (which is a simple square) is used when the letter Mem appears at the end of a word. Thus, one Mem $\square$ consists of four lines (sides of a square) and the other Mem $\Delta$ consists of 2 lines at the right and bottom and 2 modified lines at the top and left. In total the Mem consists of 8 components (6 identical and 2 slightly different). These 8 components correspond to the 8 gluons ( 6 of which have mathematically symmetrical characterizations).

3) The Weak force: the Aleph $x$ is actually a composite of 3 letters; on the top right and bottom left are 2 identical Yuds ', and separating them is 1 diagonal Vav ?. The 2 Yuds clearly represent the $\pm \mathrm{W}$ boson, and the Vav represents the $\mathrm{Z}$ boson.

\section{Particle Masses-Some Predictions}

Measured Particle masses versus predicted particle masses are illustrated in Table 2.

Column 1 of Table 2 contains the particle names (where the author has provided a descriptive name for those particles still to be discovered). The actual measured mass for each particle, with its error range, is represented in Columns 2 and 3. These mass values are obtained from the Particle Data Group 2015 update [9]. Columns 4 and 5 contain the corresponding Hebrew letters and their numerical values. Columns 6 and 7 contain a scale factor and the predicted particle masses which are obtained, and will be discussed below:

1) Matter particles: The electron, with its well defined mass, is used as the control factor to compute the matter particle masses. The electron corresponds to the letter Yud ', whose value is 10.The scale factor for level 1 (lowest energy) on the right-side pillar is the electron mass divided by 10 or 0.0511 . To obtain the scale factor for level 1 on the left-side pillar we multiply by the pillar ratio 365/248 and obtain 0.0752. Then to obtain level 2 scale factor on the left-side pillar we multiply level 1 scale factor by 365/2 and again to obtain the level 3 scale factor we multiply the level 2 scale factor by 365/2. To obtain the predicted mass for any given particle we multiply the particle value by the scale factor by (Column 5 times Column 6). All results for quarks (rows above the electron on Table 2) are within measurement error except for the bottom quark. The muon and tau masses are

\footnotetext{
${ }^{1}$ These numbers correspond to the number of commandments for the Israelites: 613 in total (or middle pillar) divided into 248 positive, e.g., give charity (associated with the right-side pillar), and 365 negative, e.g., do not kill (associated with the left-side pillar).
} 
Table 2. Particle masses.

\begin{tabular}{|c|c|c|c|c|c|c|c|c|}
\hline 1 & 2 & 3 & 4 & 5 & 6 & 7 & 8 & 9 \\
\hline \multirow[t]{2}{*}{ Particle } & Measured mass & Error/range & Letter & Letter value & Scale factor & Predicted mass & Predicted/actual & Comment \\
\hline & & (Mev/c2) & & & & (Mev/c2) & & \\
\hline Up quark & 2.3 & $+0.7,-0.5$ & lamed & 30 & 0.0752 & 2.26 & 0.981 & $<1$ sigma \\
\hline Down quark & 4.8 & $+0.5,-0.3$ & samesh & 60 & 0.0752 & 4.51 & 0.940 & $<1$ sigma \\
\hline Charm quark & 1275.0 & \pm 25 & tzadi & 90 & 13.7254 & 1235.28 & 0.969 & $<2$ sigma \\
\hline Strange quark & 95.0 & \pm 5 & zayin & 7 & 13.7254 & 96.08 & 1.011 & $<1$ sigma \\
\hline Top quark & $173,200.0$ & 176,500 & ayin & 70 & 2504.8799 & $175,341.59$ & 1.012 & In range \\
\hline Bottom quark & 4180.0 & 4660 & vav & 6 & 2504.8799 & $15,029.28$ & 3.596 & Out of range \\
\hline Electron & 0.5 & & yud & 10 & 0.0511 & 0.51 & 1.000 & Control \\
\hline Muon & 105.7 & \pm 0.0000035 & kuf & 100 & 1.0561 & 105.61 & 1.000 & Out of range \\
\hline Tau & 1776.9 & \pm 0.12 & hey & 5 & 355.4411 & 1777.21 & 1.000 & $<3$ sigma \\
\hline Dark 1 & & & pe & 80 & 13.7254 & 1098.03 & & \\
\hline Dark 2 & & & gimel & 3 & 2504.8799 & 7514.64 & & \\
\hline Dark 3 & & & kaf & 20 & 1.0561 & 21.12 & & \\
\hline Dark 4 & & & bet & 2 & 355.4411 & 710.88 & & \\
\hline Higgs boson & 125,090 & \pm 240 & resh & 200 & 626.4847 & $125,296.94$ & 1.002 & $<1$ sigma \\
\hline Higgs-like & & & dalet & 4 & $192,017.556$ & $768,070.22$ & & \\
\hline $\mathrm{Z}$ boson & $91,187.6$ & \pm 2.1 & alef-yud & & & $91,187.60$ & 1.000 & Control \\
\hline W boson & 80,385 & \pm 15 & alef-vav & & 0.8831 & $80,524.53$ & 1.002 & Out of range \\
\hline
\end{tabular}

computed in a similar way. However, an extra scale factor relating to cross letter ratios must be applied to obtain a fit to the data. At level 2 the scale factor is modified by dividing it by 6, the ratio of the letters Samesh 0 to Yud ', and at the level 3 it is further modified by dividing by 7/19, the ratio of the letters Zayin $i$ over Kuf $\supset$ (using their simple values as shown in Table 1). It is unclear why these modifications are required. The author has not worked on neutrino masses.

2) Dark particles: These same scale factors derived above are used in the same manner to predict dark particle masses. One obtains a dark matter particle around $1.1 \mathrm{GeV}$ and a dark energy partners at about $21 \mathrm{MeV}$ (of the order a tau neutrino mass?) with corresponding higher energy pairs at $7.5 \mathrm{GeV}$ and $711 \mathrm{MeV}$. As expected, from the vertical particles discussion in Section 2, the left-side dark particles are heavy (GeV's) or the dark matter particles, and the right-side dark particles are the lighter (MeV's) or dark energy particles.

3) Higgs-like particle: The scale factor for level 1 (lowest energy) on the right-side pillar is 0.0511 . To obtain the scale factor for level 1 on the middle pillar we multiply by 40 and obtain 2.044. Then to obtain level 2 scale factor on the middle pillar we multiply level 1 scale factor by 613/2 and again to obtain the level 3 scale factor we multiply the level 2 scale factor by 613/2. To obtain the predicted mass for any given particle we multiply the particle value by the scale factor by (Column 5 times Column 6). The Higgs (a level 2 particle) predicted mass is within measurement error its higher mass cousin at exactly 6.13 times the Higgs mass or $768 \mathrm{GeV}$. This compares with preliminary observations of a potential particle at around $750 \mathrm{GeV}$ (750 and 760 respectively for ATLAS and CMS) [1] [2].

4) Weak force particles: Intuitively the horizontal particles would be expected to have zero mass. We know, however, that the weak force particles have mass due to symmetry breaking. The ratio of the components of the letter Aleph $\times$ : Vav (6) over Yud ' (10); times the ratio of the pillars they connect (365/248) yields 0.883 for the ratio of the mass of $\mathrm{W}$ boson over the $\mathrm{Z}$ boson force particles. This ratio is used to predict the $\mathrm{W}$ boson mass 
from the $\mathrm{Z}$ boson mass. The predicted $\mathrm{W}$ boson mass is approximately correct but outside of measurement error.

\section{Conclusions}

The Standard Model is a triumph of physics. However, it is incomplete in several areas. In particular, it does not incorporate gravity; it does not deal with dark matter and energy particles; it does not explain why there are three levels of matter particles, and it fails to provide a relationship between the masses of most of the particles. An ancient manuscript contains a model for all building blocks of the physical world that is more complete. This model consists of 22 particles at 3 energy levels. It includes both matter particles, force particles and several gravity/mass particles which do not interact with the known forces other than with gravity. It also provides a means to calculate particle masses. Such a model sheds some light on where we might look to find more particles. Among the model's predictions are 1) a heavier Higgs-like particle at 6.13 times the Higgs mass, 2) dark matter particles at about 1.1 and $7.5 \mathrm{GeV}$, and 3) dark energy particles at around 21 and $711 \mathrm{MeV}$.

Further ongoing research is required to refine the predictions of this proposed model. However, with results coming out of the LHC it is felt that the preliminary analysis presented herein should be shared while further refinements of the proposed model continue to be developed.

\section{References}

[1] Aad, G., et al. (2015) ATLAS Collaboration, Note ATLAS-CONF-2015-081, CERN, Geneva. http://cds.cern.ch/record/2114853

[2] Khachatryan, V., et al. (2015) CMS Collaboration, Note CMS-PAS-EXO-15-004, CERN, Geneva. https://cds.cern.ch/record/2114808

[3] Kaplan, R.A. (1997) Sefer Yetzirah—The Book of Creation: In Theory and Practice. Weiser Books, San Francisco.

[4] Kaplan, R.A. (1997) Sefer Yetzirah—The Book of Creation: In Theory and Practice. Weiser Books, San Francisco, 29.

[5] Kaplan, R.A. (1997) Sefer Yetzirah-The Book of Creation: In Theory and Practice. Weiser Books, San Francisco, 145-152.

[6] Kaplan, R.A. (1997) Sefer Yetzirah-The Book of Creation: In Theory and Practice. Weiser Books, San Francisco, 197-201.

[7] Kaplan, R.A. (1997) Sefer Yetzirah-The Book of Creation: In Theory and Practice. Weiser Books, San Francisco, 159-161.

[8] Kaplan, R.A. (1997) Sefer Yetzirah-The Book of Creation: In Theory and Practice. Weiser Books, San Francisco, 145-148.

[9] Olive, K.A., et al. (2014) (Particle Data Group). Chinese Physics C, 38, Article ID: 090001. http://pdg.lbl.gov/2015/tables/contents_tables.html 